Check for updates

Cite this: RSC Adv., 2019, 9, 24791

Received 16th June 2019

Accepted 28th July 2019

DOI: $10.1039 / c 9 r a 04507 d$

rsc.li/rsc-advances

\section{Highly efficient biodegradation of reactive blue 19 under the activation of tea residue by a newly screened mixed bacterial flora DDMY $2 \uparrow$}

\author{
Xuehui Xie, (D) *ab Xiulin Zheng, ${ }^{a}$ Chengzhi Yu, ${ }^{a}$ Qingyun Zhang, ${ }^{a}$ Yiqin Wang, ${ }^{a}$ \\ Junhao Cong, ${ }^{a} \mathrm{Na} \mathrm{Liu},{ }^{c}$ Zhenjiang He, ${ }^{d}$ Bo Yang ${ }^{\star a}$ and Jianshe Liu ${ }^{\text {ab }}$
}

In this study, a newly screened mixed bacterial flora DDMY2 had high decolorization capacity for anthraquinone dye reactive blue 19 (RB19) and the decolorization efficiency of $300 \mathrm{mg} \mathrm{L}^{-1}$ RB19 could reach up to $98 \%$ within $48 \mathrm{~h}$ in the presence of tea residue. Results indicated that RB19 could be efficiently decolorized by flora DDMY2 in wide ranges of $\mathrm{pH}$ values $(5.0-9.0)$, temperatures $\left(30-40{ }^{\circ} \mathrm{C}\right)$ and initial dye concentrations $\left(50-500 \mathrm{mg} \mathrm{L}^{-1}\right)$ under the activation of tea residue. Concentration of tea residue had been proved to significantly impact the decolorization performance. UV-vis spectrophotometry, Fourier transform infrared spectrometry and liquid chromatography/time-of-flight/ mass spectrometry analysis showed three identified degradation products and the possible degradation pathway of RB19 was speculated. High-throughput sequencing analysis revealed the community structures of bacterial flora before and after domestication by tea residue. Based on the result, it was inferred that unclassified_o_Pseudomonadales, Brevibacillus, Stenotrophomonas and Bordetella activated by tea residue were responsible for the excellent decolorization performance. Results of this research deepen our understanding of the biodegradation process of anthraquinone dyes by bacterial flora and broaden the knowledge of utilizing tea residue as a bioactivator in biological treatment.

\section{Introduction}

Synthetic dyes are widely used in various industries, such as textiles, paper printing, cosmetics, plastics, food and leather. ${ }^{1}$ Among them, textile industries mainly have desizing, washing, bleaching, drying and other processes, ${ }^{2}$ which produce a large amount of effluent containing complex and refractory dyes. These dyes are discharged into water bodies, which can reduce the light penetration and oxygen transfer, resulting in changes to photosynthesis and aquatic ecosystems. ${ }^{3}$ Dyes are usually physically and chemically stable in the natural environment and therefore they are highly recalcitrant and harmful to living things. ${ }^{4}$

\footnotetext{
${ }^{a}$ State Environmental Protection Engineering Center for Pollution Treatment and Control in Textile Industry, College of Environmental Science and Engineering, Donghua University, 2999\# North Renmin Road, Songjiang District, Shanghai 201620,China.E-mail: xiexuehui@dhu.edu.cn; xiulinzxl@163.com; hyywsdklts@ 163.com; zqyfqyy@163.com; 18321195601@163.com; junhaocong@163.com; yangbo@dhu.edu.cn; liujianshe@dhu.edu.cn; Fax:+86-21-67792522

${ }^{b}$ Shanghai Institute of Pollution Control and Ecological Security, Shanghai 200092, P. R. China

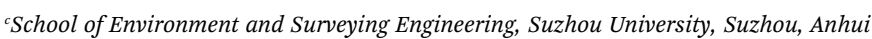
234000, China. E-mail: liuna900301@163.com

${ }^{d}$ School of Metallurgy and Environment, Central South University, Changsha 410083, P. R. China.E-mail: hzjcsu@csu.edu.cn

$\dagger$ Electronic supplementary information (ESI) available. See DOI: $10.1039 / \mathrm{c} 9 \mathrm{ra} 04507 \mathrm{~d}$
}

Given the refractory dye-containing wastewater, numerous physical and chemical treatment methods have positive effects on the removal of such wastewater. However, high cost and secondary pollution have greatly constrained these processes. ${ }^{5}$ Alternatively, biological treatment methods have been paid more attention, which have advantages of low cost, simple operation and environmental friendliness. ${ }^{6,7}$ At present, a vast literature on biological treatments using bacteria, fungi and algae have been reported to efficiently decolorize azo dyes. ${ }^{\mathbf{8 - 1 0}}$ Unfortunately, the study on biodecolorization of anthraquinone dyes is still insufficient and the effect is undesirable. For instance, Holkar et al. ${ }^{\mathbf{1 1}}$ isolated a strain of Escherichia sp. F NCIM 5545, which just decolorized $50 \mathrm{mg} \mathrm{L}^{-1}$ reactive blue 19.

Herein, tea residue as a domestic waste biomass, was employed to boost the decolorization performance of anthraquinone dye reactive blue 19 (RB19) by a newly screened mixed bacterial flora DDMY2. The impacts of several parameters (namely, pH, temperature, initial dye concentration and tea residue concentration) on decolorization were systematically investigated. UV-Vis spectrophotometry, Fourier transform infrared spectrometry (FTIR) and liquid chromatography/timeof-flight/mass spectrometry (LC-TOF-MS) were used to detect the biodegradation products of RB19. In addition, highthroughput sequencing revealed the microbial community structures of initial flora DDMY2 (not domesticated by tea residue) and flora DDMY2 (domesticated by tea residue for 12 
months). The possible functional bacterial groups activated by tea residue were also speculated. These findings can provide new insights and extend the knowledge of biodegradation towards anthraquinone dyes. Moreover, this study can enlarge the utilization of waste biomass tea residue in industrial wastewater treatment.

\section{Materials and methods}

\subsection{Chemicals and culture medium}

Commercial grade reactive blue 19 (CAS No. 2580-78-1, MW $626.54, \lambda_{\max }=596 \mathrm{~nm}$ ) was purchased from Sigma-Aldrich (USA). All other chemicals and reagents were of analytical grade, except for dichloromethane (HPLC level). The culture medium (CM medium) consisted of $\mathrm{NH}_{4} \mathrm{Cl}\left(0.20 \mathrm{~g} \mathrm{~L}^{-1}\right), \mathrm{Na}_{2} \mathrm{SO}_{4}$ $\left(0.50 \mathrm{~g} \mathrm{~L}^{-1}\right), \mathrm{KH}_{2} \mathrm{PO}_{4}\left(2.66 \mathrm{~g} \mathrm{~L}^{-1}\right)$ provided from Sinopharm Chemical Reagent (Shanghai) Co., Ltd. and yeast extract $(3.00 \mathrm{~g}$ $\mathrm{L}^{-1}$ ) obtained from Sangon Biotech (Shanghai) Co., Ltd. Tea residue was made from West Lake Longjing tea (Hangzhou, China) which was repeatedly brewed at $80{ }^{\circ} \mathrm{C}$ until tea soup became colorless. The culture medium and tea residue were sterilized under the condition of $121^{\circ} \mathrm{C}, 0.12 \mathrm{MPa}$ for $20 \mathrm{~min}$.

\subsection{Screening of RB19 decolorizing bacterial flora DDMY2}

RB19 decolorizing bacterial flora DDMY2 was screened from activated sludge taken from Songjiang sewage treatment plant (Songjiang, Shanghai, China). Through continuously increasing the dye concentration in the CM medium, activated sludge suspension $(10 \%, \mathrm{v} / \mathrm{v})$ was used to decolorize $300 \mathrm{mg} \mathrm{L}^{-1} \mathrm{RB} 19$ until the decolorization rate reached more than $70 \%$ (ESI $\dagger$ Text S1). The obtained dye decolorizing bacterial flora was named as DDMY2.

\subsection{Experiments on enhancement effect of tea residue}

In order to investigate the enhancement effect of tea residue, eight groups were carried out and they were dye + distilled water (DD), dye + medium (DM), dye + distilled water + flora DDMY2 (DDM), dye + medium + flora DDMY2 (DMF), medium (M), dye + medium + tea residue (DMT), dye + distilled water + flora DDMY2 + tea residue (DDFT), dye + medium + flora DDMY2 + tea residue (DMFT), respectively. The inoculation of flora DDMY2 was $10 \%(\mathrm{v} / \mathrm{v})$ and the culture was incubated at $37{ }^{\circ} \mathrm{C}$ under static condition. After regular intervals for $24 \mathrm{~h}$, aliquots $(2 \mathrm{~mL})$ were collected and centrifuged at $6200 \times g$ for $10 \mathrm{~min}$. Subsequently, the obtained supernatant was analyzed by UV-vis spectrophotometer (IMPLEN, German). Decolorization rates were measured according to the method described by Xie et al. ${ }^{12}$

\subsection{Decolorization assay}

The capacity of decolorizing RB19 by bacterial flora DDMY2 at varying conditions was assessed. The culture media were amended/incubated with different $\mathrm{pH}$ values (4.0-9.0), temperatures $\left(30-45{ }^{\circ} \mathrm{C}\right)$ and initial dye concentrations (50$\left.500 \mathrm{mg} \mathrm{L}^{-1}\right)$, respectively. Different concentrations of tea residue had also been chosen $\left(0-6 \mathrm{~g} \mathrm{~L}^{-1}\right)$ to investigate the enhancement effect. If not specified, concentration of tea residue and $\mathrm{RB} 19$ are respective $3 \mathrm{~g} \mathrm{~L}^{-1}$ and $300 \mathrm{mg} \mathrm{L}^{-1}$. The inoculation, culture conditions and the method for determination of decolorization rates were the same as those in Section 2.3 .

\subsection{Biodegradation products analysis}

The culture medium containing RB19 and its decolorizing solution were harvested and centrifuged at $6200 \times g$ for $10 \mathrm{~min}$ to obtain the supernatant. Afterwards, the supernatant was scanned through UV-vis spectrophotometry. FTIR analysis was performed to determine the changes in functional groups of dye before and after decolorization. The preparation steps are shown in ESI $\dagger$ Text S2. Prepared samples were measured on NICOLET 6700 spectrophotometer (Thermo, America) in the mid-IR region of $600-4000 \mathrm{~cm}^{-1}$ with 16 -scan speed at room temperature. Besides, the biodegradation products after decolorization were also analyzed by liquid chromatography/time-offlight/mass spectrometry (LC-TOF-MS, Agilent QTOF6520, USA). The test information is shown in ESI $\uparrow$ Text S3.

\subsection{High-throughput sequencing analysis}

Microbial community structures of initial flora DDMY2 (not domesticated by tea residue, sample initial-DDMY2) and flora DDMY2 (domesticated by tea residue for 12 months) were investigated via high-throughput sequencing method reported by Xie et $a .^{12}$ The specific experimental procedures are mentioned in ESI $\uparrow$ Text S4 and all sequences had been deposited in the NCBI GenBank with accession no. SRP132025.

\subsection{Statistical analysis}

All experiments and measurements were conducted in triplicate. All data were shown as the means \pm standard deviations. The Student " $t$ " test or one-way analysis of variance (ANOVA) was used to evaluated significant differences of two levels of a single factor or more than two levels of a single factor, respectively. The level $p$-value $\leq 0.05$ was regarded to be statistically significant and the level $p$-value $\leq 0.01$ was considered to be highly significant.

\section{Results and discussion}

\subsection{Decolorization performance of flora DDMY2}

It took 150 generations (12 months) to possess the decolorizing bacterial flora DDMY2 through the method of dye gradient pressure domestication. The obtained flora DDMY2 had efficient decolorization performance of RB19 (300 $\mathrm{mg} \mathrm{L}^{-1}$ ) and its efficiency reached up to $98 \%$ with tea residue after 48 h. From Fig. 1, it was obvious that the dark blue colored dye changed to light yellow-brown in the presence of flora DDMY2 and tea residue (group A3), speculating RB19 was decolorized which might be caused by either adsorption or degradation. Comparing group A1 (medium + tea residue) with group A2 $(\mathrm{RB} 19+$ medium + tea residue), both of them showed no significant color change ( $p$-value $=0.241)$ and the color removal of group A2 after $48 \mathrm{~h}$ was only $2.52 \pm 0.56 \%$, indicating the adsorption of tea residue could be negligible. The group A4 with 
(a)
A1

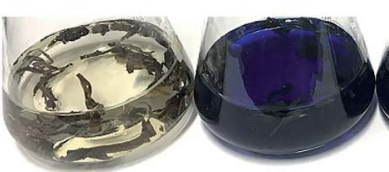

A1

A2 (b)

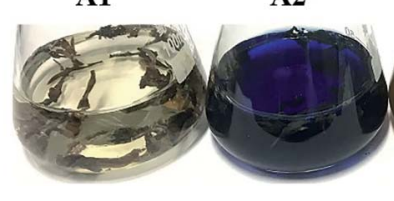

A3

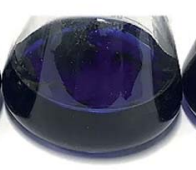

A3
A4

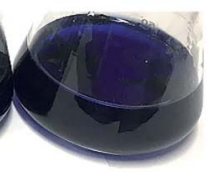

A4
Fig. 1 Images of $300 \mathrm{mg} \mathrm{L}^{-1}$ RB19 before (a) and after (b) decolorization by flora DDMY2 under the activation of tea residue. A1 represented medium with tea residue, A2 represented medium with tea residue and RB19, A3 represented medium with tea residue, RB19 and flora DDMY2, A4 represented medium with tea residue, RB19 and heat-inactivated flora DDMY2.

heat-inactivated flora DDMY2 (inactivated in a high-pressure steam sterilization pot at $121{ }^{\circ} \mathrm{C}, 0.12 \mathrm{MPa}$ for $20 \mathrm{~min}$ ), medium, tea residue and RB19 was just $6.54 \pm 1.09 \%$ of decolorization after $48 \mathrm{~h}$. Thus, the contribution of abiotic process (e.g. chemical degradation and adsorption of heatinactivated flora DDMY2) for RB19 removal can be excluded. ${ }^{\mathbf{1 3}}$ Summing up the above, the decolorization of RB19 was attributed to the biodegradation by flora DDMY2 with tea residue.

\subsection{Enhancement effect of tea residue}

The confirmatory study on the enhancement effect of tea residue towards RB19 decolorization by flora DDMY2 was carried out. From Fig. 2a, it showed that only the group DMFT (dye + medium + flora DDMY2 + tea residue) was notably decolorized from dark blue to light orange, followed by group DMF (dye + medium + flora DDMY2), which was lightly decolorized to light blue. The difference between these two groups was due to the addition of tea residue ( $p$-value $=0.003$ ). Combined with Fig. 2b, group DMFT had the highest decolorization rate of $92.18 \pm 0.98 \%$ after $48 \mathrm{~h}$, followed by group DMF $(67.23 \pm 0.82 \%)$. The $24.95 \%$ increase demonstrated tea residue played a vital role in boosting decolorization and had a strong enhancement effect. Decolorization rates of group DDF (dye + distilled water + flora DDMY2) and group DDFT (dye + distilled water + flora DDMY2 + tea residue) were $9.22 \pm 0.79 \%$ and $36.19 \pm 0.75 \%$, respectively. It proved that flora DDMY2 could be also promoted by tea residue without any other nutrients ( $p$-value $=0.002$ ), but the effect was limited. Compared with group DMF (dye + medium + flora DDMY2), group DM (dye + medium) had the decolorization rate of $0.28 \pm$ $0.07 \%$, suggesting decolorization required the action of flora DDMY2 and it made a highly significant contribution ( $p$-value $<$ 0.01 ). In addition, group DMT (dye + medium + tea residue) was only with decolorization rate of $2.83 \pm 0.58 \%$, manifesting tea residue could not be used as adsorbent to remove RB19 as described in Section 3.1. This finding was different from existing research that tea residue was used as adsorbent to remove dyes. ${ }^{\mathbf{1 4 , 1 5}}$ Based on our previous work, through single factor and orthogonal experimental analysis of common substances in tea residue, epigallocatechin gallate was deciphered to be the key active component which was responsible for the enhancement effect on the decolorization performance by activating the functional bacteria. ${ }^{16}$

\subsection{Effects of physico-chemical parameters on decolorization performance of DDMY2}

3.3.1. Effect of $\mathbf{p H}$. Compared to optimum pH 5-7, extreme pH can severely inhibit microbial activities and even cause death. ${ }^{17}$ In this work, flora DDMY2 could adapt to a wide range of $\mathrm{pH}$ in the presence of tea residue and it showed better decolorization performance with the increase of $\mathrm{pH}$ value from 6.5 to 9.0 (Fig. 3a). The maximum decolorization rate was observed at $\mathrm{pH} 7.0(95.83 \pm 1.11 \%$ of decolorization rate, $p$ value $<0.01$, which was similar to the result reported by Chen et $a{ }^{18}$ When $\mathrm{pH}$ value was lower than 7.0 presented acidic condition, the decolorization rate rapidly decreased. In
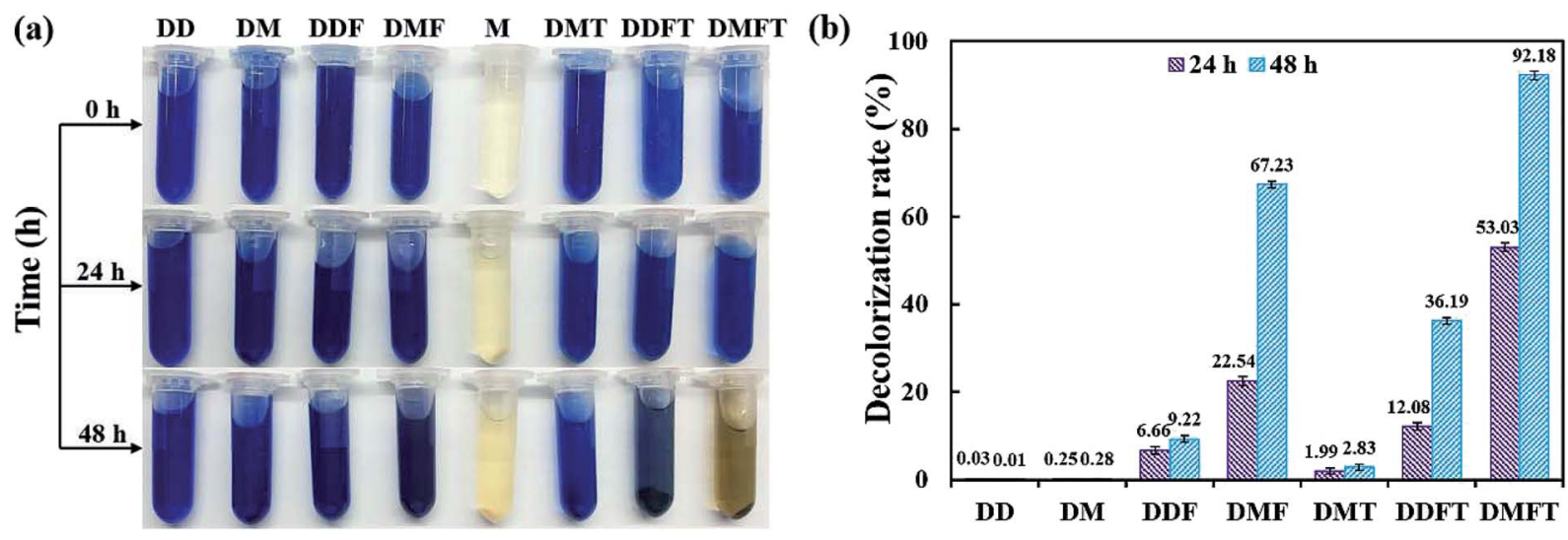

Fig. 2 Images of enhancement effect of tea residue on RB19 decolorization by flora DDMY2 (a) and decolorization rates of tea residue towards RB19 by flora DDMY2 (b). DD: dye + distilled water; DM: dye + medium; DDF: dye + distilled water + flora DDMY2; DMF: dye + medium + flora DDMY2; M: medium; DMT: dye + medium + tea residue; DDFT: dye + distilled water + flora DDMY2 + tea residue; DMFT: dye + medium + flora DDMY2 + tea residue. Group M had no decolorization rate, so it was not included in the histogram. 
(a)

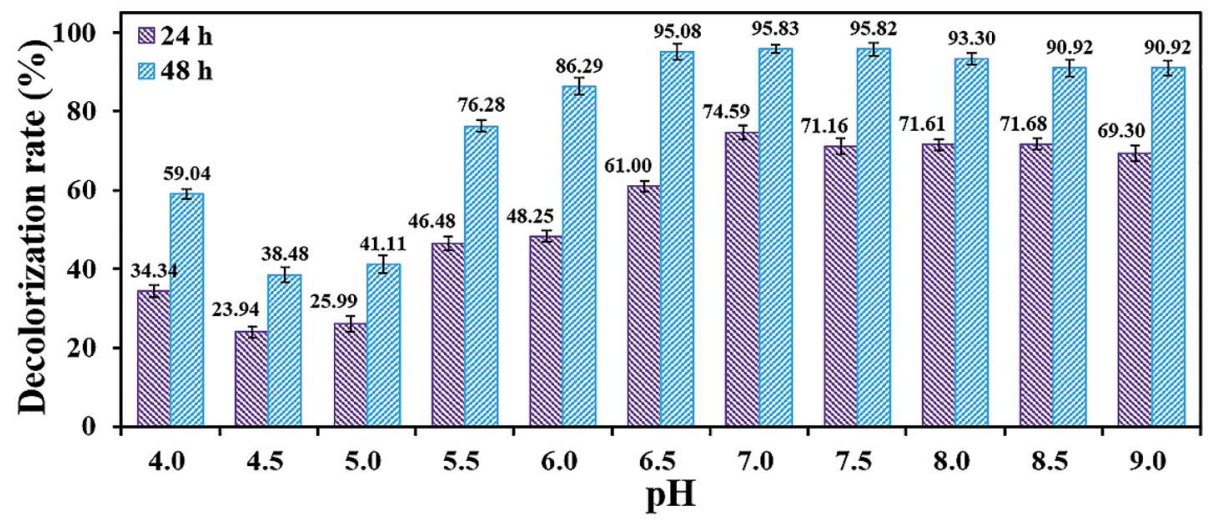

(b)

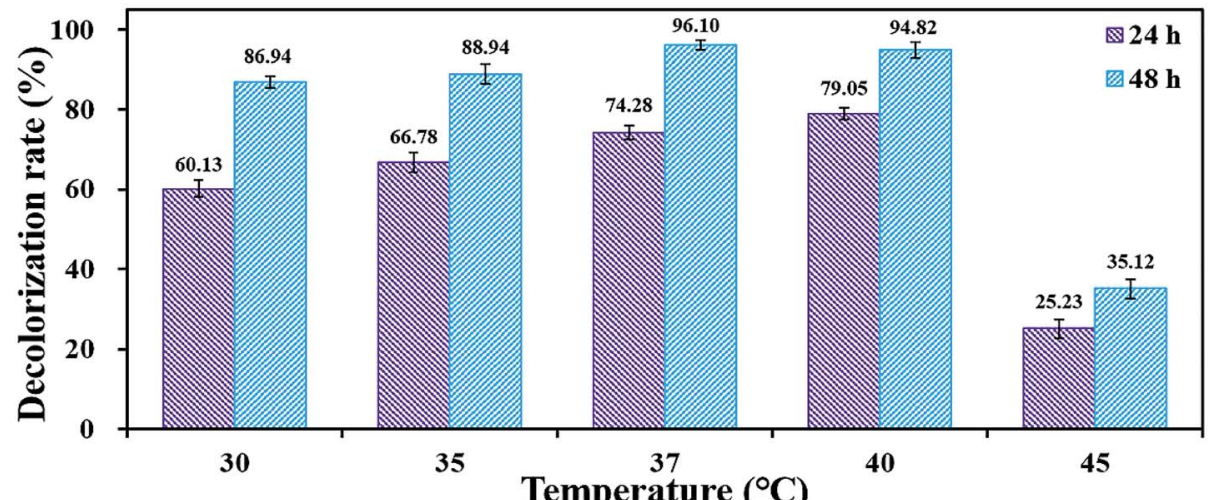

(c)
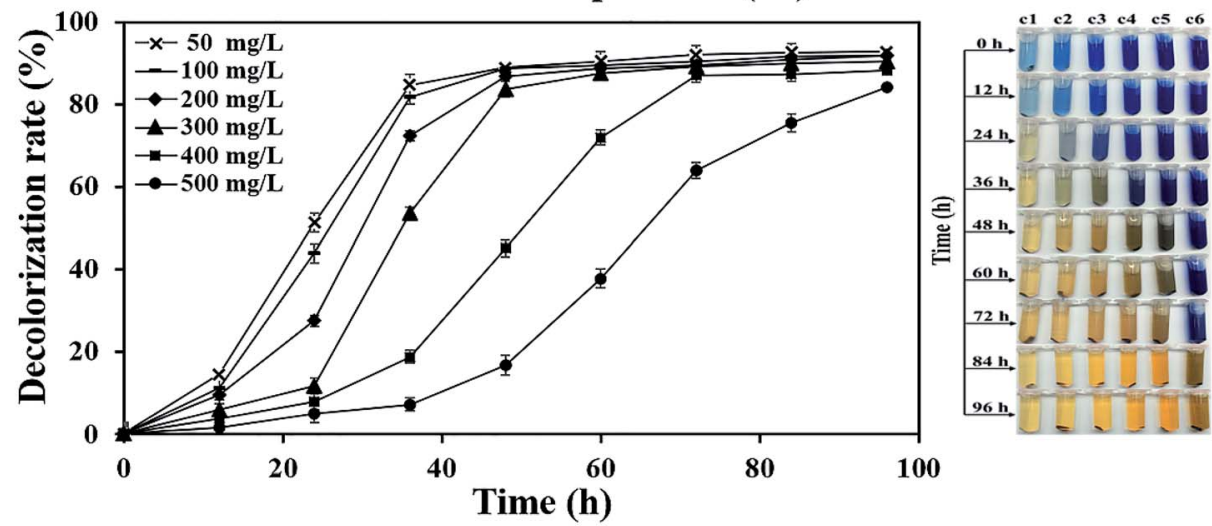

(d)
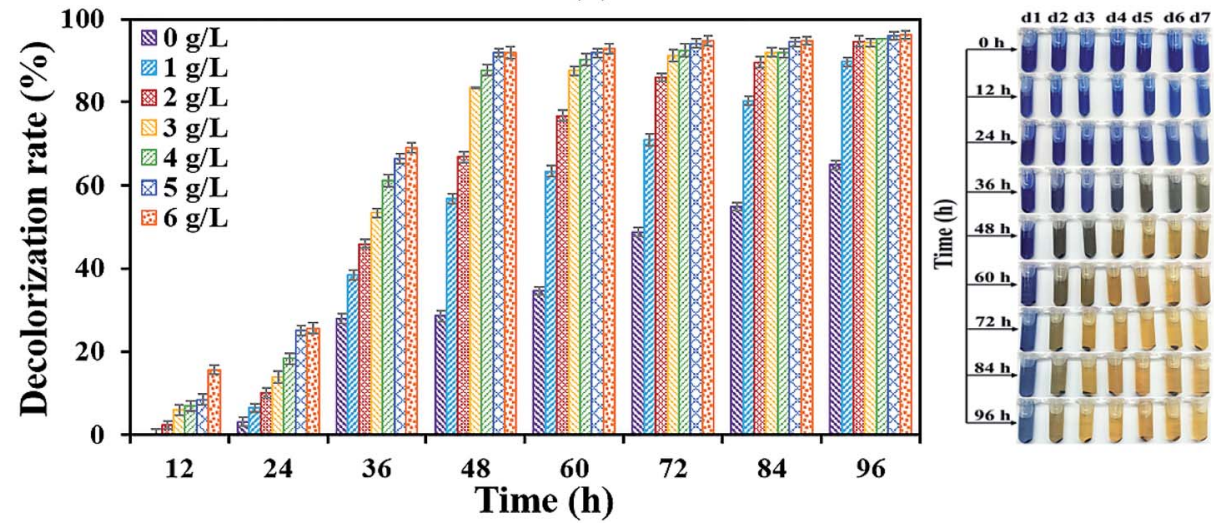

Fig. 3 Decolorization potentiality of flora DDMY2 under the activation of tea residue at different pH values (a), temperatures (b), initial dye concentrations (c) and concentrations of tea residue (d). The right part of (c) and (d) were images of decolorization samples with different initial dye concentrations and different concentrations of tea residue. Serial numbers from c1 to c6 represented initial dye concentration $50-$ $500 \mathrm{mg} \mathrm{L}^{-1}, \mathrm{~d} 1$ to $\mathrm{d} 7$ represented concentrations of tea residue $0-6 \mathrm{~g} \mathrm{~L}^{-1}$, respectively. 
addition, flora DDMY2 also exhibited considerable decolorization effect under neutral and alkaline conditions. The $\mathrm{pH}$ value from neutral to weakly alkaline had already been found to have the maximum decolorization of different dyes. ${ }^{19-21}$ The reason might be that the neutral and weakly alkaline conditions were suitable for the growth of flora DDMY2. Moreover, it was related to the enzymatic system involved in the degradation process. For instance, some functional enzymes (e.g. laccase) of dye decolorizing microbes are usually stable at alkaline conditions. ${ }^{22}$

3.3.2. Effect of temperature. Temperature is an important factor affecting the biological process of dye degradation. Under the activation of tea residue, flora DDMY2 displayed the best decolorization efficiency towards RB19 at $37{ }^{\circ} \mathrm{C}(96.10 \pm 1.31 \%$ of decolorization rate, $p$-value $<0.01$ ), followed by $40{ }^{\circ} \mathrm{C}$ and $35{ }^{\circ} \mathrm{C}$ (Fig. 3b). This phenomenon was coincided with the result that dye decolorization was presented at the most suitable temperature of $35{ }^{\circ} \mathrm{C}$ and $37{ }^{\circ} \mathrm{C}^{23}$ Wang et al. ${ }^{24}$ also discovered range of $27-37^{\circ} \mathrm{C}$ was the optimum decolorization temperature of reactive red by bacterial strain Citrobacter sp. CK3. The temperature value higher or lower than this range $\left(37-40{ }^{\circ} \mathrm{C}\right)$ was found obviously reduce the decolorization capacity of flora DDMY2 in this study. For example, after $48 \mathrm{~h}$, the decolorization rate only achieved $35.12 \pm 2.31 \%$ at $45{ }^{\circ} \mathrm{C}$, which decreased about $60 \%$ as compared to that at $37{ }^{\circ} \mathrm{C}$ ( $p$-value $\left.=0.002\right)$. In addition, through measuring $\mathrm{OD}_{600}$ and extracellular quinone reductase activity (involved in the degradation of RB19) at different temperatures, it was found that at $48 \mathrm{~h}$, the biggest $\mathrm{OD}_{600}(3.24 \pm 0.02)$ and maximum enzymatic activity $(34.27 \pm$ $1.35 \mu \mathrm{M}$ of NADH reduced $\mathrm{min}^{-1} \mathrm{mg}^{-1}$ of protein) were at $37^{\circ} \mathrm{C}$ (data not shown), which was consistent with the maximum decolorization rate. Hence, it was considered that the changes in cell viability or bacterial enzymatic activity on the grounds of different temperatures caused the changes in decolorization performance towards RB19 by flora DDMY2 under the activation of tea residue..$^{25}$

3.3.3. Effect of initial dye concentration. In actual wastewater treatment process, the dye concentration is usually serious and has certain inhibitory effect on microorganisms. ${ }^{26}$ As a consequence, initial dye concentration was set from $50 \mathrm{mg} \mathrm{L^{-1 }}$ to $500 \mathrm{mg} \mathrm{L}^{-1}$ and the decolorization effect was shown in Fig. 3c. The decolorization curves stated that, after $48 \mathrm{~h}$, the decolorization performance remained stable $(>80 \%)$ when RB19 was not exceeding $300 \mathrm{mg} \mathrm{L}^{-1}$ ( $p$-value $\left.=0.314\right)$. However, when the concentration was more than $400 \mathrm{mg} \mathrm{L}^{-1}$, it still reached higher decolorization efficiency but took a longer time (96 h) to adapt the pressure derived from high dye concentration. $^{27,28}$ This suggested that flora DDMY2 had outstanding potential to decolorize high concentration of RB19 and was relatively better than other bacterial strains under the activation of tea residue. ${ }^{\mathbf{1 1 , 2 9}}$ The phenomenon was similar to the result founded by $\mathrm{Fu}$ et al. $^{30}$ Moreover, even if the decolorization efficiency decreased with increasing dye concentration, the actual moles of RB19 removed were increased as a function of RB19 concentration. For example, at 48 h, $88.69 \pm 1.80 \%$ decolorization of $100 \mathrm{mg} \mathrm{L}^{-1} \mathrm{RB} 19$ was equivalent to $88.69 \pm$ $1.80 \mathrm{mg} \mathrm{L}^{-1}$ of dye removed; however, $83.73 \pm 1.57 \%$ decolorization of $300 \mathrm{mg} \mathrm{L}^{-1} \mathrm{RB} 19$ was equivalent to $251.19 \pm$ $4.71 \mathrm{mg} \mathrm{L}^{-1}$ of dye removed. This case was also mentioned by Holkar et al. $^{31}$ in the biodegradation process of RB19 by Klebsiella sp. C NCIM 5546.

3.3.4. Effect of tea residue concentration. Different concentrations of tea residue had obvious influence on the decolorization performance by flora DDMY2. For the first $48 \mathrm{~h}$, with the increase of tea residue concentration, the decolorization rate increased observably ( $p$-value $<0.01$, Fig. $3 \mathrm{~d}$ ). However, when tea residue concentration exceeded $3 \mathrm{~g} \mathrm{~L}^{-1}$, the promotion of increasing concentration was not so obvious from $48 \mathrm{~h}$ to $96 \mathrm{~h}$ ( $p$-value $>0.05)$. The reason for this phenomenon might be: (i) the promotion for flora DDMY2 had reached the maximum limitation; (ii) many residual antimicrobial substances in tea residue accumulated and then offset the enhancement effect. From the changes of apparent color, it was observed that as tea residue concentration increased from $0 \mathrm{~g} \mathrm{~L}^{-1}$ to $6 \mathrm{~g} \mathrm{~L}^{-1}$, the color changed significantly from dark blue to light yellow. Based on these results, the optimum concentration of tea residue for activating decolorization was suggested as $3 \mathrm{~g} \mathrm{~L}^{-1}$ in this study.

\subsection{Analysis of biodegradation products}

The UV-vis spectra full-wavelength scanning results of the decolorizing solution during the decolorization process were collected. Fig. 4 a showed that from $0 \mathrm{~h}$ to $48 \mathrm{~h}$, a peak centered at $596 \mathrm{~nm}$ decreased, which was the characteristic absorption peak representing RB19. Especially, at $48 \mathrm{~h}$, the characteristic peak intensity almost completely disappeared. Simultaneously, two new absorption peaks centered at $465 \mathrm{~nm}$ and $485 \mathrm{~nm}$ appeared and amplified gradually. Finally, they reached the highest intensity at $48 \mathrm{~h}$. After another $48 \mathrm{~h}$, the newly generated peaks centered at $465 \mathrm{~nm}$ and $485 \mathrm{~nm}$ began to decrease, demonstrating these intermediates were further degraded by flora DDMY2. Xu et al. ${ }^{32}$ reported that bacteria degraded dyes into intermediates which were further transformed into final products.

The FTIR spectra of RB19 differed significantly before and after decolorization, which indicated there was biodegradation in the decolorization process (Fig. 4b). The spectrum of $0 \mathrm{~h}$ showed the prominent absorption peak at $3393.2 \mathrm{~cm}^{-1}$ for stretching vibration of $\mathrm{N}-\mathrm{H}$ on $-\mathrm{NH}_{2}$, which was the primary amino group in RB19. ${ }^{33}$ As decolorization proceeded, the peak $3393.2 \mathrm{~cm}^{-1}$ decreased significantly and disappeared completely after $48 \mathrm{~h}$, indicating that the primary amino group $-\mathrm{NH}_{2}$ converted to secondary amino group $-\mathrm{NH}$. The peaks at $2921.7 \mathrm{~cm}^{-1}$ and $2850.3 \mathrm{~cm}^{-1}$ represented the symmetry and asymmetry of $\mathrm{C}-\mathrm{H}$ on alkane $\left(-\mathrm{CH}_{2}-\right)$ stretching vibration and their intensity became higher after $48 \mathrm{~h}$. The peak $1665.3 \mathrm{~cm}^{-1}$ was the $\mathrm{C}=\mathrm{O}$ stretching vibration, which represented the vibration of the carbonyl group in the anthraquinone structure of RB19. It decreased gradually over time, indicating the anthraquinone group was degraded in this process. The peak $1454.1 \mathrm{~cm}^{-1}$ was the deformation vibration of $\mathrm{C}-\mathrm{H}$ on $-\mathrm{CH}_{2}$. The peak at $1260.8 \mathrm{~cm}^{-1}$ represented $\mathrm{C}-\mathrm{N}$ stretching vibration on the benzene ring. The peaks at $1095.4 \mathrm{~cm}^{-1}$ and $1022.6 \mathrm{~cm}^{-1}$ represented $\mathrm{S}=\mathrm{O}$ stretching vibration, ${ }^{34}$ which came from the 
(a)

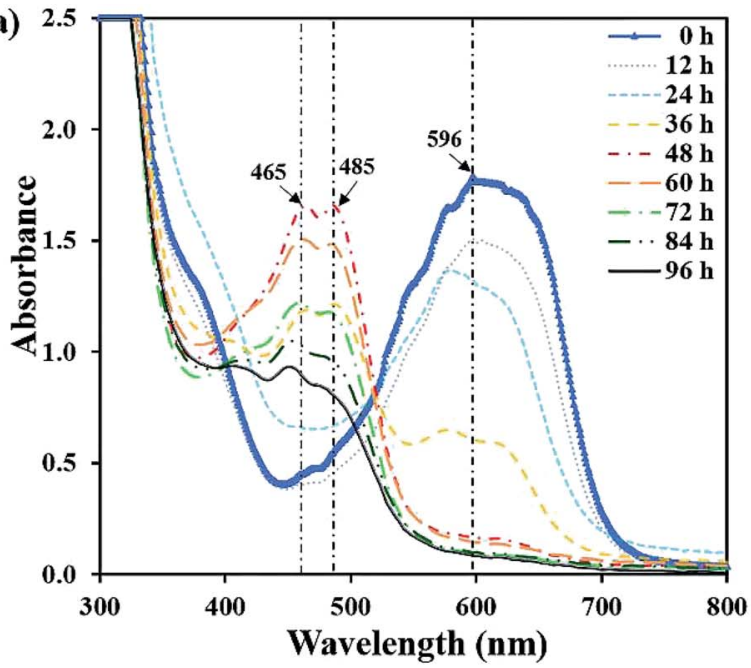

(b)

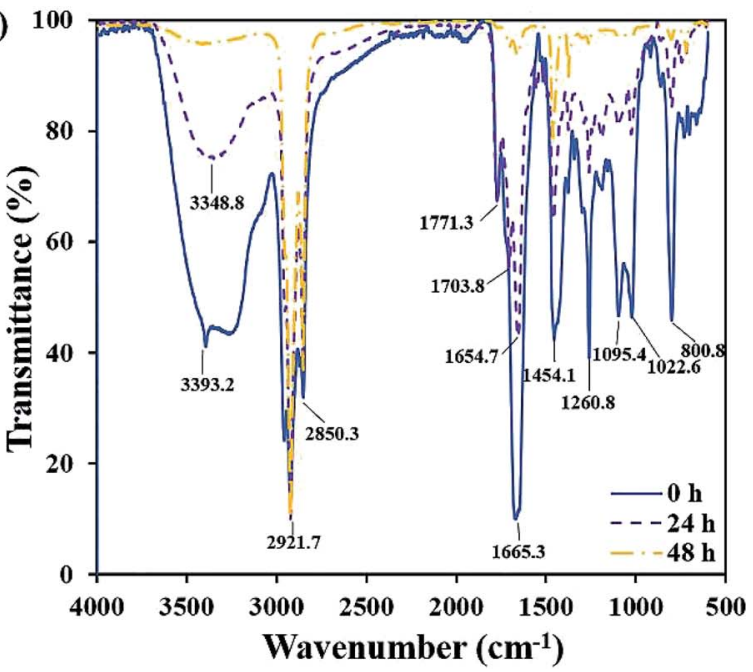

Fig. 4 UV-vis spectra full-wavelength scanning of the decolorization process (a), and FTIR spectra at different time of the decolorization process (b).

sulfoxide group of RB19. These decreased and absent absorption peaks revealed bonds between anthraquinone ring and benzene ring were broken and RB19 had been decomposed by flora DDMY2, thereby greatly improved the biodegradability, which was beneficial for further treatment.

The products came from flora DDMY2 decolorizing RB19 for $48 \mathrm{~h}$ were analyzed by LC-TOF-MS. From these results, a total of three major metabolites were identified in the positive and negative ion modes. The first product was detected in positive ion mode, presenting the peak retention time (14.629 $\mathrm{min})$ and the mass-to-charge ratio $(\mathrm{m} / \mathrm{z} 102.1200)$ (Fig. S1a-c $\dagger$ ). Compared the mass spectrum with the NIST library, it was confirmed to be hexan-1-amine with a matching degree of $85.89 \%$ (Table 1), which was decomposed from RB19 by the biochemical action of flora DDMY2. The amines came from RB19 decolorization were also found by Vasconcelos et $a l .{ }^{35}$ In the negative ion mode, the separation effect was obvious and there were four distinct ion peaks which were detected mainly concentrated in 9-10 min (Fig. S1d†). The peaks retention time of $10.020 \mathrm{~min}$ and $9.458 \mathrm{~min}$ (Fig. S1e and $\mathrm{f} \dagger$ ) corresponded to $\mathrm{m} / \mathrm{z} 156.9900$ and 194.9500, respectively (Fig. S1g and $h \dagger$ ). The former was identified as benzenesulfonate ion with a matching degree of $90.01 \%$ and the latter was regarded as 3,6-dihydroxyphthalic acid with a matching degree of $83.50 \%$ (Table 1), which was formed by the quinone ring opening. Products of similar structures were often reported, such as dibutyl phthalate and 2,6-di-tert-butyl-4 methylphenol were detected by Castillo and Barcelo. ${ }^{36}$

Combined UV-Vis, FTIR and LC-TOF-MS analysis results, the chemical transformation process of RB19 by flora DDMY2 under the activation of tea residue was deduced in Fig. 5. The

Table 1 Products of RB19 after $48 \mathrm{~h}$ decolorization identified by LC-TOF-MS analysis

Dye $\begin{aligned} & \begin{array}{l}\text { Peak retention } \\ \text { time }(\mathrm{min})\end{array} \\ & \begin{array}{c}\text { Mass-to-charge } \\ \text { ratio }(\mathrm{m} / \mathrm{z})\end{array}\end{aligned}$




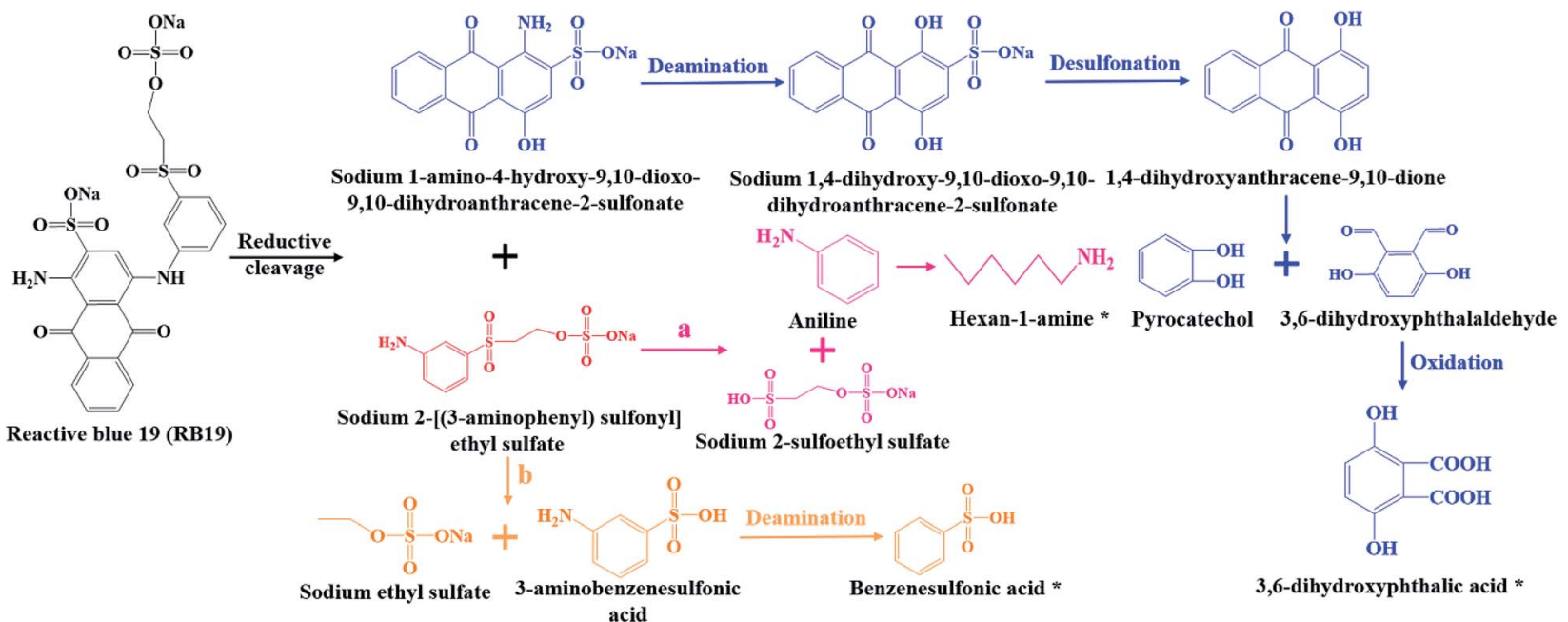

Fig. 5 Derivation of molecular transformation mechanism of RB19 by bacterial flora DDMY2 under the activation of tea residue. (a) and (b) were two possible metabolic pathways, the symbol $(*)$ represented the detected products.

dye RB19 was directly hydrolyzed into sodium 1-amino-4hydroxy-9,10-dioxo-9,10-dihydroanthracene-2-sulfonateand and sodium 2-[(3-aminophenyl) sulfonyl] ethyl sulfate. The former was determinated by Bilal et al. ${ }^{37}$ in photo-assisted catalytic degradation of RB19. The latter was also revealed by Fanchiang and Tseng ${ }^{38}$ in degradation of RB19. Subsequently, the amino (a)

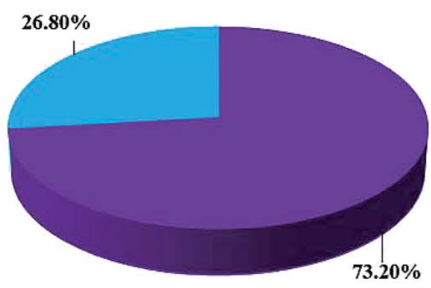

initial-DDMY2

(b)

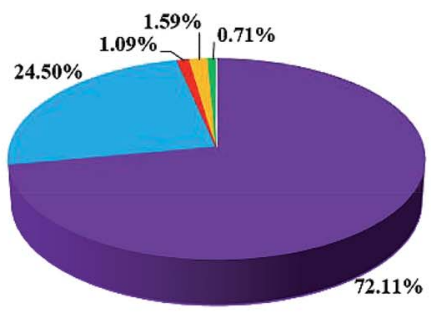

initial-DDMY2

(c)

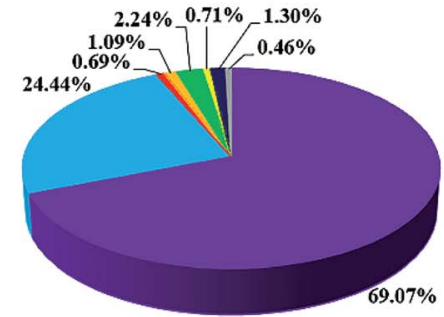

initial-DDMY2

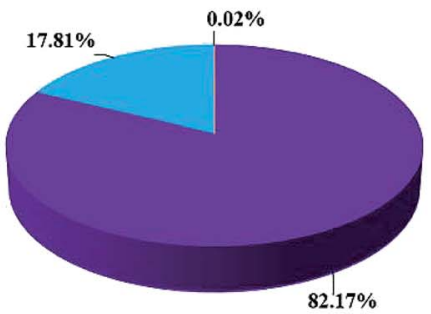

DDMY2

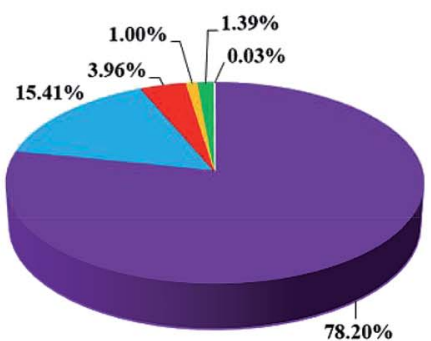

DDMY2
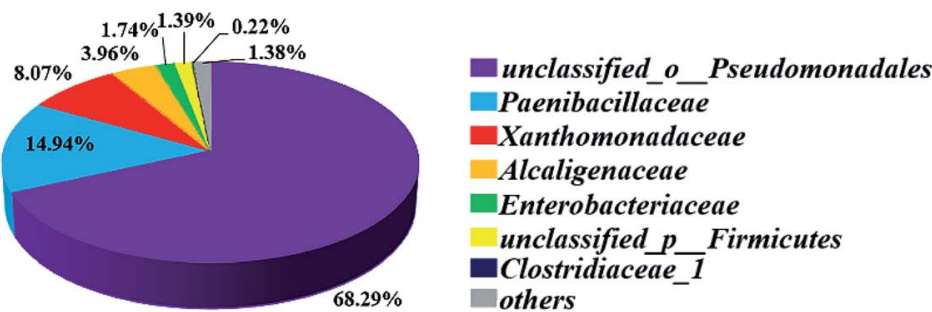

DDMY2
- Proteobacteria

- Firmicutes

others
Gammaproteobacteria

Bacilli

Betaproteobacteria

Clostridia

unclassified_p_Firmicutes

others

Fig. 6 Microbial community structures of sample initial-DDMY2 and sample DDMY2 on different classification levels: phylum level (a); class level (b); family level (c), and the group others represented the sum of the level percentage less than $1 \%$. 
(a)

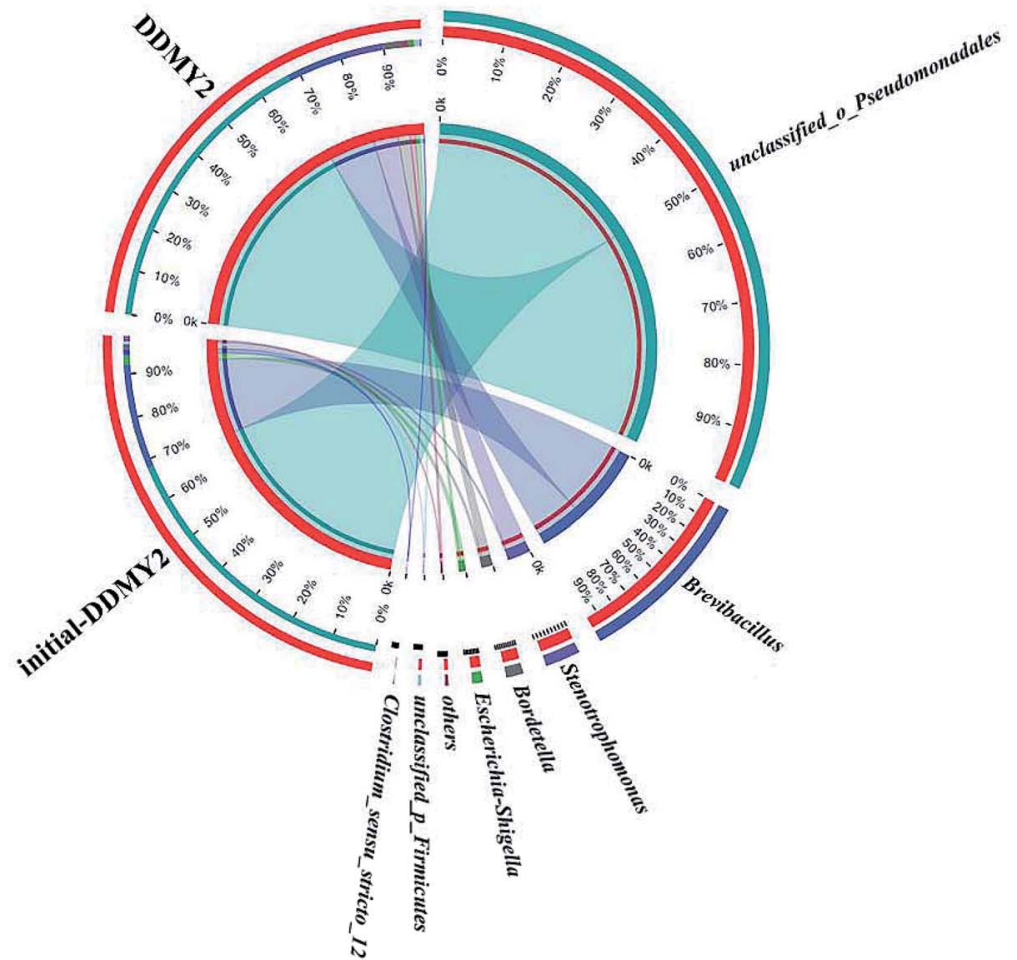

(b)

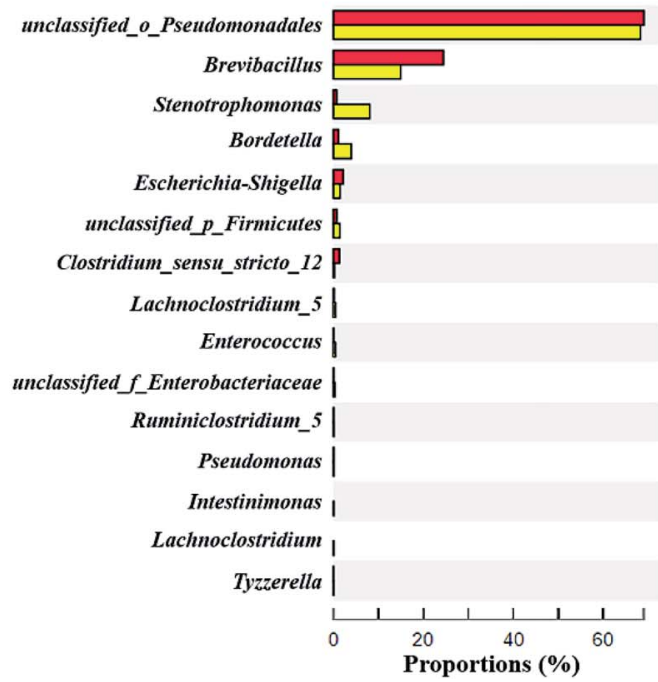

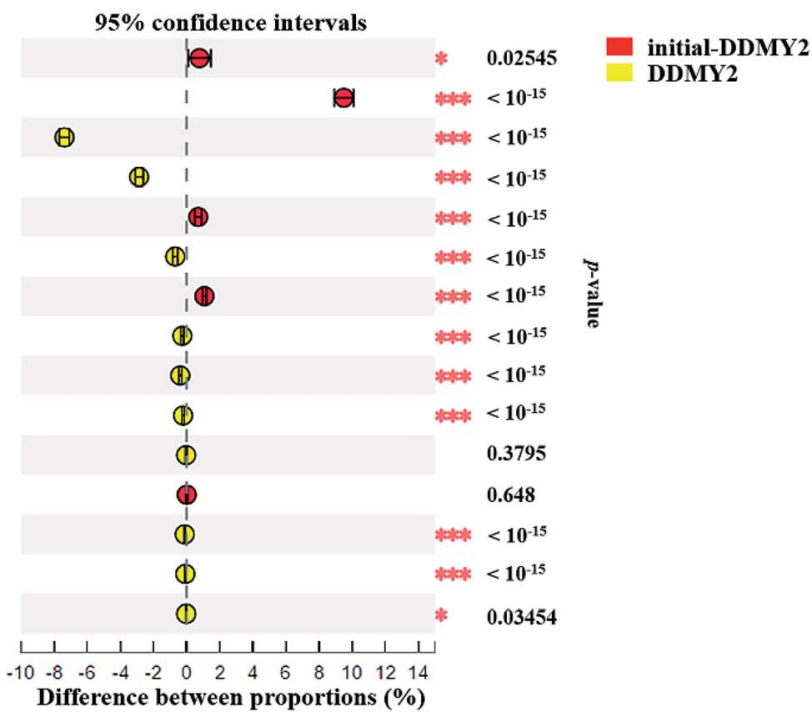

Fig. 7 Microbial community distribution of sample initial-DDMY2 and sample DDMY2 at genus level (a), among group others represented the sum of the level percentage less than $1 \%$, and the width of the bars from genus level indicated the relative abundance in each sample. The Fisher' exact test bar plot on genus level between sample initial-DDMY2 and sample DDMY2 (b), among the column length corresponding to the genus indicated the relative abundance in each sample, and the right part represented the $p$-value $(* 0.01<p \leq 0.05 ; * * 0.001<p \leq 0.01 ; * * * p \leq$ 0.001). Significance level: 0.05. Multiple check correction: False Discovery Rate.

group of sodium 1-amino-4-hydroxy-9,10-dioxo-9,10dihydroanthracene-2-sulfonateand transformed into hydroxy group by hydrolysis or aromatic substitution to form sodium 1,4dihydroxy-9,10-dioxo-9,10-dihydroanthracene-2-sulfonate, which was certified by McCallum et $a .^{39}$ Then, the 1,4dihydroxyanthracene-9,10-dione formed by desulfonation, which was detected by Ghazalian et al. $^{40}$ in visible light photocatalytic degradation of RB19. With the process going on, the quinone ring further opened and formed pyrocatechol and 3,6-dihydroxyphthalaldehyde. The pyrocatechol was shown in the electroFenton process for degradation of RB19. ${ }^{41}$ The 3,6-dihydroxyphthalaldehyde was oxidized to 3,6-dihydroxyphthalic acid which had been detected in this study. For sodium 2-[(3-aminophenyl) sulfonyl] ethyl sulfate, it could be seen that there might be two possible metabolic pathways. In one way, sodium 2-[(3aminophenyl) sulfonyl] ethyl sulfate was hydrolyzed into 
aniline and sodium 2-sulfoethyl sulfate, where aniline was found by He et $a .^{42}$ in mineralization of RB19 by ozonation combined with sonolysis. The benzene ring of aniline was further opened and converted to hexan-1-amine found in this work. In the other way, sodium 2-[(3-aminophenyl) sulfonyl] ethyl sulfate might be hydrolyzed into 3-aminobenzenesulfonic acid and sodium ethyl sulfate. Subsequently, 3-aminobenzenesulfonic acid was transformed into benzenesulfonic acid by deamination, which was discovered in this research. The whole degradation pathway of RB19 by flora DDMY2 under the activation of tea residue was firstly described in this paper.

\subsection{Microbial community structure analysis}

High-throughput sequencing analysis was used to reveal the microbial community structures of sample intial-DDMY2 and sample DDMY2, which produced 40066 and 38685 sequence tags, respectively. Of these produced sequence tags, 34628 reads were randomly selected for further study. In addition, a total of 24 and 33 OTUs were individually obtained from initial-DDMY2 and DDMY2 with clustering at a 3\% dissimilarity level. The rarefaction curve can be used to compare the richness, uniformity or diversity of samples and indicate whether the reads of samples are reasonable. In this study, the Sobs rarefaction curves became relatively flat with the increase of sequences (Fig. S2a †), indicating the results were reasonable and exact. $^{43}$ Based on the Shannon rarefaction curves (Fig. S2b $\dagger$ ), sample DDMY2 had the higher community diversity.

In order to further analyze the community composition of sample initial-DDMY2 and sample DDMY2 at different taxonomic levels, sequences were divided into different taxonomic classifications through RDP classifier. On classification of phylum level (Fig. 6a), two phyla Proteobacteria and Firmicutes were detected separately in two samples, which were frequently discovered in the dye biodegradation systems. ${ }^{\mathbf{4 4 , 4 5}}$ Proteobacteria was richer than Firmicutes in two samples and the proportion of Proteobacteria increased under the activation of tea residue, indicating Proteobacteria might be enriched by tea residue and played vital roles in RB19 biodegradation. On the class level (Fig. 6b), Gammaproteobacteria $(72.11 \%$ and $78.20 \%$, respectively) was the absolutely dominant class in sample initial-DDMY2 and sample DDMY2, followed by Bacilli $(24.50 \%$ and $15.41 \%$, respectively), Betaproteobacteria (1.09\% and $3.96 \%$, respectively), etc. Gammaproteobacteria was widely presented in the hydrolytic acidification process of dyeing wastewater treatment. ${ }^{\mathbf{4 6}}$ Bacilli and Betaproteobacteria were also reported to exist in simulated printing wastewater reactors. ${ }^{\mathbf{4 3}}$ Further analysis was carried out to reveal the microbial community abundance on the family level (Fig. 6c). The unclassified_o_Pseudomonadales was the predominant family in initialDDMY2 and DDMY2, which occupied $69.07 \%$ and $68.29 \%$, respectively. The proportions were nearly no change. However, the classes with greater abundance variation in initial-DDMY2 and DDMY2 were Paenibacillaceae $(24.44 \%$ and $14.94 \%$, respectively) and Xanthomonadaceae (0.69\% and $8.07 \%$, respectively).

The Circos map showed the profiles of genus in sample initial-DDMY2 and sample DDMY2. As shown in Fig. 7a, seven genera accounting for more than 1\% were detected. Among them, unclassified_o_Pseudomonadales made up the largest proportions $(69.07 \%$ and $68.29 \%$, respectively) of the total effective sequences in sample initial-DDMY2 and sample DDMY2, followed by Brevibacillus $(24.44 \%$ and $14.94 \%$, respectively), Stenotrophomonas (0.69\% and $8.07 \%$, respectively) and Bordetella $(1.09 \%$ and $3.96 \%$, respectively). It has been found that the consortium of bacteria, including Brevibacillus, was used to decolorize textile industry effluent. ${ }^{47}$ What's more, Stenotrophomonas had also been reported to have the ability to decolorize azo dye Xylene Fast Yellow 2G. ${ }^{48}$ In order to more intuitively observe the changes in the proportions of genera belonging to sample initial-DDMY2 and sample DDMY2, the Fisher' exact test bar plot was shown in Fig. 7b. Compared initial-DDMY2 with DDMY2, the percentages of unclassified_o_Pseudomonadales, Brevibacillus, Escherichia-Shigella, Clostridium_sensu_stricto_12 and Pseudomonas in sample DDMY2 decreased. The decreasing amplitudes of them were not significant (except for Brevibacillus), and unclassified_o_Pseudomonadales and Brevibacillus might play a certain role in decolorization because of their lager proportions. However, Stenotrophomonas and Bordetella increased remarkably (increased by $7.38 \%$ and $2.87 \%$, respectively), which related to the enhancement effect of tea residue. The remaining eight genera only showed a slight increase. Hence, it was speculated that unclassified_o_Pseudomonadales, Brevibacillus, Stenotrophomonas and Bordetella might be mainly responsible for decolorizing RB19 by DDMY2 under activation of tea residue.

\section{Conclusions}

Bacterial flora DDMY2 possessed high decolorization performance towards RB19 under the activation of tea residue. Flora DDMY2 activated by tea residue could efficiently decolorize RB19 under conditions with wide ranges of $\mathrm{pH}$ values, temperatures and initial dye concentrations. Concentration of tea residue also had significant effect on decolorization performance. UV-vis, FTIR and LC-TOF-MS results indicated RB19 was degraded into small organics and the possible degradation pathway was speculated. High-throughput sequencing results revealed the changes in the community structures of initial-DDMY2 and DDMY2, of which unclassified_o_Pseudomonadales, Brevibacillus, Stenotrophomonas and Bordetella might be activated by tea residue. These findings could provide new insights of biodegradation process of anthraquinone dyes by bacterial flora, and broaden our knowledge of utilization of tea residue in the treatment of industrial dyeing wastewater.

\section{Conflicts of interest}

There are no conflicts to declare. 


\section{Acknowledgements}

The authors acknowledge the financial support by the Fundamental Research Funds for the Central Universities (2232018G11, 2232019D3-22), the National Key Research and Development Program of China (Grant No. 2016YFC0400501), the Graduate Student Innovation Fund of Donghua University (CUSF-DH-D2019078), Anhui Provincial Natural Science Foundation (1808085QE176), the scientific research program of Anhui Provincial Education Department (KJ2018A0444) and the Suzhou University Startup Foundation for Doctor (2016jb04), the "Chenguang Program" supported by Shanghai Education Development Foundation and Shanghai Municipal Education Commission (No. 16CG40). This work was partially supported by Shanghai Leading Academic Discipline Project (B604).

\section{References}

1 J. H. Franco, B. F. da Silva, R. V. Oliveira, G. Meireles, D. P. de Oliveira, A. A. de Castro, T. C. Ramalho and M. V. B. Zanoni, Sci. Total Environ., 2018, 613, 1093-1103.

2 A. B. dos Santos, F. J. Cervantes and J. B. van Lier, Bioresour. Technol., 2007, 98, 2369-2385.

3 Q. Zhang, X. Xie, Y. Liu, X. Zheng, Y. Wang, J. Cong, C. Yu, N. Liu, J. Liu and W. Sand, Bioresour. Technol., 2019, 280, 430-440.

4 N. d. C. Lima Beluci, G. A. Pisano Mateus, C. S. Miyashiro, N. C. Homem, R. G. Gomes, M. R. Fagundes-Klen, R. Bergamasco and A. M. Salcedo Vieira, Sci. Total Environ., 2019, 664, 222-229.

5 C. Wu, Y. Zhou, X. Sun and L. Fu, Environ. Sci. Pollut. Res., 2018, 25, 8315-8329.

6 T. Ito, Y. Adachi, Y. Yamanashi and Y. Shimada, Water Res., 2016, 100, 458-465.

7 K. Vikrant, B. S. Giri, N. Raza, K. Roy, K.-H. Kim, B. N. Rai and R. S. Singh, Bioresour. Technol., 2018, 253, 355-367.

8 A. Ghosh, M. G. Dastidar and T. R. Sreekrishnan, Int. Biodeterior. Biodegrad., 2017, 119, 448-460.

9 E. J. R. Almeida and C. R. Corso, Int. J. Environ. Sci. Technol., 2019, 16, 1319-1328.

10 K. Behl, S. Sinha, M. Sharma, R. Singh, M. Joshi, A. Bhatnagar and S. Nigam, Chem. Eng. J., 2019, 364, 552561.

11 C. R. Holkar, A. B. Pandit and D. V. Pinjari, Bioresour. Technol., 2014, 173, 342-351.

12 X. Xie, N. Liu, J. Ping, Q. Zhang, X. Zheng and J. Liu, Chemosphere, 2018, 201, 578-585.

13 Y.-N. Liu, F. Zhang, J. Li, D.-B. Li, D.-F. Liu, W. W. Li and H.-Q. Yu, Environ. Sci. Technol., 2017, 51, 8616-8623.

14 B. Naraghi, F. Zabihi, M. R. Narooie, M. Saeidi and H. Biglari, Electronic Physician, 2017, 9, 4312-4321.

15 C. S. Patil, D. B. Gunjal, V. M. Naik, N. S. Harale, S. D. Jagadale, A. N. Kadam, P. S. Patil, G. B. Kolekar and A. H. Gore, J. Cleaner Prod., 2019, 206, 407-418.

16 X. Zheng, X. Xie, C. Yu, Q. Zhang, Y. Wang, J. Cong, N. Liu, Z. He, B. Yang and J. Liu, Chemosphere, 2019, 233, 110-119.
17 S. Sang, J. D. Lambert, C.-T. Ho and C. S. Yang, Pharmacol. Res., 2011, 64, 87-99.

18 C.-C. Chen, C.-Y. Chen, C.-Y. Cheng, P.-Y. Teng and Y.-C. Chung, J. Hazard. Mater., 2011, 196, 166-172.

19 A. U. Chaudhari, D. Paul, D. Dhotre and K. M. Kodam, Water Res., 2017, 122, 603-613.

20 K. Rybczynska-Tkaczyk, A. Swiecilo, K. A. Szychowski and T. Kornillowicz-Kowalska, Ecotoxicol. Environ. Saf., 2018, 147, 776-787.

21 E. Rahimi, Y. D. Shahamat, B. Kamarehei, A. Zafarzadeh and M. R. Khani, Int. J. Environ. Sci. Technol., 2018, 15, 19411948.

22 C. Zhang, H. Diao, F. Lu, X. Bie, Y. Wang and Z. Lu, Bioresour. Technol., 2012, 126, 80-86.

23 S. Hussain, Z. Maqbool, S. Ali, T. Yasmeen, M. Imran, F. Mahmood and F. Abbas, Ecotoxicol. Environ. Saf., 2013, 98, 331-338.

24 H. Wang, J. Q. Su, X. W. Zheng, Y. Tian, X. J. Xiong and T. L. Zheng, Int. Biodeterior. Biodegrad., 2009, 63, 395-399.

25 F. Mahmood, M. Shahid, S. Hussain, T. Shahzad, M. Tahir, M. Ijaz, A. Hussain, K. Mahmood, M. Imran and S. A. K. Babar, Bioresour. Technol., 2017, 235, 176-184.

26 Z. Wang, Q. Yin, M. Gu, K. He and G. Wu, J. Hazard. Mater., 2018, 357, 226-234.

27 C. R. Holkar, A. J. Jadhav, D. V. Pinjari, N. M. Mahamuni and A. B. Pandit, J. Environ. Manage., 2016, 182, 351-366.

28 R. Liu, X. Lu, Q. Tian, B. Yang and J. Chen, Desalination, 2011, 271, 287-294.

29 J. Krishnan, A. A. Kishore, A. Suresh, B. Madhumeetha and D. G. Prakash, Int. Biodeterior. Biodegrad., 2017, 119, 16-27.

30 L. Fu, Y.-N. Bai, Y.-Z. Lu, J. Ding, D. Zhou and R. J. Zeng, J. Hazard. Mater., 2019, 364, 264-271.

31 C. R. Holkar, H. Arora, D. Halder and D. V. Pinjari, Int. Biodeterior. Biodegrad., 2018, 133, 194-201.

$32 \mathrm{M}$. Xu, X. Zhong, W. Cao, J. Guo, Y. Cen and G. Sun, Microbiology., 2005, 32, 5-9.

33 Z. Khan, K. Jain, A. Soni and D. Madamwar, Int. Biodeterior. Biodegrad., 2014, 94, 167-175.

34 N. Liu, X. Xie, B. Yang, Q. Zhang, C. Yu, X. Zheng, L. Xu, R. Li and J. Liu, Environ. Sci. Pollut. Res., 2017, 24, 252-263.

35 V. M. Vasconcelos, F. L. Migliorini, J. R. Steter, M. R. Baldan, N. G. Ferreira and M. R. d. V. Lanza, J. Environ. Chem. Eng., 2016, 4, 3900-3909.

36 M. Castillo and D. Barcelo, Anal. Chim. Acta, 2001, 426, 253264.

37 M. Bilal, T. Rasheed, H. M. N. Iqbal, C. Li, H. Wang, H. Hu, W. Wang and X. Zhang, Chem. Eng. Res. Des., 2018, 129, 384390.

38 J.-M. Fanchiang and D.-H. Tseng, Chemosphere, 2009, 77, 214-221.

39 J. E. B. McCallum, S. A. Madison, S. Alkan, R. L. Depinto and R. U. R. Wahl, Environ. Sci. Technol., 2000, 34, 5157-5164.

40 E. Ghazalian, N. Ghasemi and A. R. Amani-Ghadim, J. Mol. Catal. A: Chem., 2017, 426, 257-270.

41 Z. He, C. Gao, M. Qian, Y. Shi, J. Chen and S. Song, Ind. Eng. Chem. Res., 2014, 53, 3435-3447. 
42 Z. He, L. Lin, S. Song, M. Xia, L. Xu, H. Ying and J. Chen, Sep. Purif. Technol., 2008, 62, 376-381.

43 X. Xie, N. Liu, B. Yang, C. Yu, Q. Zhang, X. Zheng, L. Xu, R. Li and J. Liu, Int. Biodeterior. Biodegrad., 2016, 111, 14-21.

44 K. H. Balapure, K. Jain, S. Chattaraj, N. S. Bhatt and D. Madamwar, J. Hazard. Mater., 2014, 279, 85-95.

45 F. Pan, Y. Yu, A. Xu, D. Xia, Y. Sun, Z. Cai, W. Liu and J. Fu, J. Hazard. Mater., 2017, 340, 36-46.
46 Q. Yang, J. Wang, H. Wang, X. Chen, S. Ren, X. Li, Y. Xu, H. Zhang and X. Li, Bioresour. Technol., 2012, 117, 155-163. 47 M. B. Kurade, T. R. Waghmode, J.-Q. Xiong, S. P. Govindwar and B.-H. Jeon, J. Cleaner Prod., 2019, 213, 884-891.

48 W. Feng, P. Song, Y. Zhang and Z. Dong, in Advances in Applied Biotechnology, VOL II, ed. T. C. Zhang and M. Nakajima, 2015, vol. 333, pp. 109-116. 